\title{
Implementation of mild therapeutic hypothermia for post-resuscitation care of sudden cardiac arrest survivors in cardiology units in Poland
}

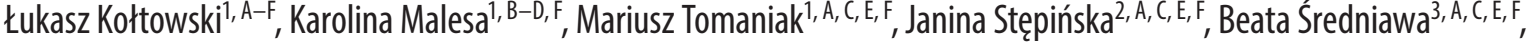

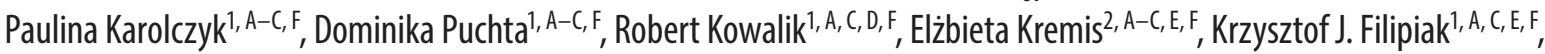 \\ Marek Banaszewski², A, B, E, F, Grzegorz Opolski ${ }^{1}$ A, C, E, F, Marta Bagińska ${ }^{1, D}$ \\ ${ }^{1} 1^{\text {st }}$ Department of Cardiology, Medical University of Warsaw, Poland \\ ${ }^{2}$ Intensive Cardiac Care Clinic, Institute of Cardiology, Warszawa, Poland \\ ${ }^{3}$ Department of Cardiology, Congenital Heart Diseases and Electrotherapy, Medical University of Silesia, Silesian Center for Heart Diseases, Zabrze, Poland \\ A - research concept and design; B - collection and/or assembly of data; C - data analysis and interpretation; \\ $D$ - writing the article; $E$ - critical revision of the article; $F$ - final approval of the article
}

\begin{abstract}
Address for correspondence
Łukasz Kołtowski

E-mail: lukasz@koltowski.com

Funding sources

None declared

\section{Acknowledgements}

The authors of this manuscript would like to thank all the participating centers who answered the questionnaire, dedicated their time and shared data regarding the local use of therapeutic hypothermia. The authors are also grateful to the Proper Medical Writing agency for providing language help and proofreading the article.
\end{abstract}

\section{Conflict of interest}

None declared

Received on December 23, 2015

Reviewed on September 14, 2016

Accepted on November 14, 2016

\begin{abstract}
Background. The post-cardiac arrest (CA) period is often associated with secondary damage of the brain that leads to severe neurological deficits. The current practice guidelines recommend the use of therapeutic hypothermia (TH) to prevent neurological deficit and improve survival.

Objectives. The aim of the study was to investigate the implementation of medical guidelines in clinical practice and to evaluate the barriers for implementation of TH in cardiology units in Poland.

Material and methods. A telephone survey, fax and online inquiry form were used to assess the implementation of TH in cardiology units in the management of unconscious patients after cardiac arrest (CA). The questions addressed the local practice, TH protocol, reasons for not using TH and outcomes of CA patients.

Results. We obtained information from 79 units out of 150 asked (53\%). At the time of the survey, 24 units (30.8\%) were using $\mathrm{TH}$ as part of their post-CA management. Of all CA patients, $45 \%$ underwent TH in cardiac intensive care units (CICU), 37.5\% in the coronary care unit (CCU) and $12.5 \%$ in the intensive care unit (ICU). The major barrier for the implementation of TH declared by the non-cooling centers was lack of sufficient knowledge regarding the technique and protocol, as well as experience (37\%); access to dedicated equipment was not perceived as an obstacle.

Conclusions. The number of cardiology units that provide TH for comatose CA patients is low. The main limiting factor for wider use of $\mathrm{TH}$ is lack of knowledge and experience. There is a clear need for urgent educational activities for cardiology units. The benefits of TH still have not reached their potential in cardiology units.
\end{abstract}

Key words: cardiac arrest, therapeutic hypothermia, resuscitation, hypothermia registry, target temperature management

DOI

10.17219/acem/66992

Copyright

Copyright by Author(s)

This is an article distributed under the terms of the

Creative Commons Attribution Non-Commercial License

(http://creativecommons.org/licenses/by-nc-nd/4.0/) 


\section{Introduction}

Cardiac arrest (CA) remains the major cause of sudden death; each year it affects around 375,000 people in Europe. Recovery from cardiac arrest with a delayed return of spontaneous circulation (ROSC) of more than $5 \mathrm{~min}$ is usually associated with an anoxic neurologic injury and is recognized as an important factor for increased morbidity and mortality. ${ }^{1}$ Therapeutic hypothermia (TH) is a well-established method which has shown a positive impact on these outcomes. ${ }^{2-4}$ The European Society of Cardiology strongly recommends the use of $\mathrm{TH}$ early after resuscitation of CA patients presenting acute myocardial infarction with ST-segment elevation (class I, evidence B) who are comatose or under deep sedation. ${ }^{5}$ Similarly, the recent European Resuscitation Council and European Society of Intensive Care Medicine Guidelines for Post-Resuscitation Care 2015 recommend active cooling of patients after CA. ${ }^{6}$ It should be underlined that $\mathrm{TH}$ is one of many treatment methods used along the resuscitation care chain and should not preclude implementation of other strategies. Furthermore, in order to ensure a high quality of care and safety, all teams providing $\mathrm{TH}$ must undergo dedicated training on the cooling protocol and TH methods.

The last survey, conducted in 2010, before European Society of Cardiology (ESC) guidelines were published, showed that the proportion of intensive care units (ICU) using TH in Poland was only $21.7 \% .^{7}$ The ICUs are usually managed by anesthesiologists, whereas patients after CA are often admitted to the cardiac intensive care units (CICU) and treated by cardiologists, therefore it is important to assure that access to $\mathrm{TH}$ management is also available in these units. At present, it is unknown what percentages of cardiology centers have a dedicated $\mathrm{TH}$ program in place and are able to offer it to their patients in everyday practice. To investigate this matter, and to identify the main barriers for the implementation of a $\mathrm{TH}$ program, we have conducted a survey among cardiology centers that treat patients after CA.

$\mathrm{TH}$, defined as controlled cooling of patients after cardiac arrest to a temperature between 32 and $34^{\circ} \mathrm{C}$ for a period of at least $24 \mathrm{~h}$, aims to temper the post-cardiac arrest syndrome inflammatory cascade. One of the underlining mechanisms that provide beneficial effects is the reduction of release of excitatory amino acids and free radicals, which terminate activating programmed cell death pathways. Moreover, the intracellular consequences of excitotoxin exposure are minimized. Furthermore, hypothermia influences cerebral blood volume, cerebral metabolic rate of oxygen and intracranial pressure, which are all decreased. As a consequence, oxygen demand and the function of the brain might be improved. ${ }^{1}$

In recent years, the development of $\mathrm{TH}$ programs across Poland has been actively supported by the Polish Cardiac Society, which, among others, included the foundation of the Polish National Registry of Therapeutic Hypothermia. In January 2014, the National Health Fund announced a dedicated reimbursement code for $\mathrm{TH}$ that ultimately eliminated the financial barriers for this method. The growing number of scientific reports on the clinical benefits of $\mathrm{TH}$ increases the awareness of this method among Polish cardiologists. ${ }^{8-11}$ Positive results are more likely to be achieved if strict criteria for inclusion and exclusion are applied.

The primary aim of the survey was to determine the implementation of the ESC and ERS guidelines in the clinical practice of cardiology units.

\section{Material and methods}

The data was prospectively collected from June to December 2014, using telephone survey, fax, online and printed inquiry form (Supplement 1). The investigators made an attempt to contact each of the 150 CICUs located in Poland. The answers were obtained from members of the CICU medical team, in particular the head of the department or designated physician. In case there was no answer to the initial email/letter with the questionnaire, the center was contacted by phone. In total, up to 3 attempts were made to contact each center. A detailed questionnaire investigated the experience of the center with $\mathrm{TH}$ (questions $1-4)$, the local practice in place for the management of the patient (question 5), type of the mild TH protocol (questions 6-8 and 11-14), mode of cooling and temperature control (questions 9-10), obstacles related to the employment of TH in clinical practice (questions 15-16) and educational training received by the unit (questions 17-19). The investigators collected declarative data on the number of patients that were managed at each CICU and those successfully discharged from the hospital.

Descriptive statistics were used to present the results; the categorical data was presented as percentage and nominal numbers. The percentages were reported in reference to the number of centers that provided the answers unless clearly stated otherwise.

\section{Results}

Out of the 150 CICUs approached in the survey, approx. half provided answers to the questionnaire $(53 \%, \mathrm{n}=79)$. Most centers replied to the questionnaire sent by traditional post (49\%, 38), 36\% (28) provided answer after telephone contact from the investigators, $16.5 \%$ (13) submitted their answers via email.

Twenty-four centers confirmed the active use of $\mathrm{TH}$ in CA patients, $63 \%$ of them declared using hypothermia in a pre-hospital setting (Table 1). Almost half of the centers cooled less than 5 CA patients per year (Fig. 1). All treating centers have a dedicated $\mathrm{TH}$ protocol in place; nearly 
Fig. 1. Therapeutic hypothermia usage per year

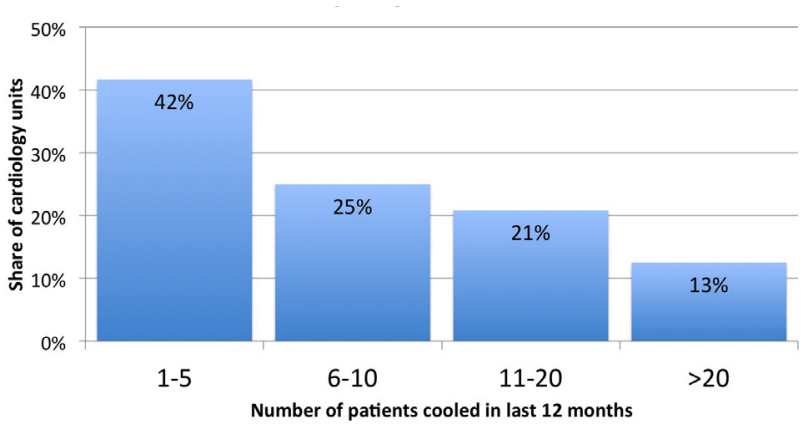

all $(96 \%, \mathrm{n}=23)$ used the target temperature range from 32 to $34^{\circ} \mathrm{C}$. In most cases $(58 \%, \mathrm{n}=14)$, the target temperature was maintained for $12-24 \mathrm{~h}$, and in $38 \%$ it lasted for more than $24 \mathrm{~h}$. The induction phase was usually performed using an intravenous infusion of cold fluids (96\%, $\mathrm{n}=23$ ). External cooling methods were applied more often $(75 \%, \mathrm{n}=18)$, usually without control equipment $(42 \%, n=10)$. Every $4^{\text {th }}$ center was equipped with an intravascular cooling system. The comparison of the main features of the $\mathrm{TH}$ protocols revealed that patients were qualified for the procedure regardless of the type of the $1^{\text {st }}$ cardiac rhythm (ventricular fibrillation, ventricular tachycardia, asystole or pulseless electrical activity).

Among the centers that do not perform TH, only 33\% ( $\mathrm{n}=18$ ) are considering using $\mathrm{TH}$ in the future as part of the local protocol for management of post CA patients. The main barrier for starting a TH program is lack of knowledge and experience $(37 \%, \mathrm{n}=20)$. The centers were not aware that dedicated $\mathrm{TH}$ educational training is available $(96 \%, n=22)$ (Table 2$)$. Only 37\% ( $n=20)$ of the centers had participated as least once in a dedicated $\mathrm{TH}$ workshop on the risks and benefits related to the procedure (Table 3).

\section{Discussion}

The extended data surveillance conducted between June and December 2014 that included 150 CICUs in Poland revealed that $30.3 \%$ (24) of units use TH in their daily practice. This data is complimentary to the previously conducted survey among Polish ICUs, which revealed that $21.7 \%$ of the anesthesiology units surveyed have a TH program in place. ${ }^{7}$ A direct comparison of this data with the present study has some clear limitations and should not be used to draw firm conclusions as the profile of the patients treated in ICUs and CICUs differs; there has been over 5 years' time difference, and meanwhile the reimbursement of TH has been introduced and there has been much more clinical evidence supporting temperature control in CA patients published recently. Still, the observed differences in the applied cooling methods show that over the years there was an increase in the usage
Table 1. Procedural characteristics and implementation of $\mathrm{TH}$ in units that routinely perform $\mathrm{TH}(\mathrm{n}=24)$

\begin{tabular}{|c|c|}
\hline \multicolumn{2}{|l|}{ Type of unit performing TH procedure } \\
\hline cardiac intensive care unit & $50 \%(12 / 24)$ \\
\hline coronary intensive unit & $12.5 \%(3 / 24)$ \\
\hline intensive care unit & $37.5 \%(9 / 24)$ \\
\hline \multicolumn{2}{|l|}{ Split of units $(n=24)$ according to: } \\
\hline \multicolumn{2}{|l|}{ The target temperature used } \\
\hline $32-34^{\circ} \mathrm{C}$ & $95.8 \%(23 / 24)$ \\
\hline$<32^{\circ} \mathrm{C}$ & $0 \%(0 / 20)$ \\
\hline$>34^{\circ} \mathrm{C}$ & $4.2 \%(1 / 24)$ \\
\hline \multicolumn{2}{|c|}{ The duration of the maintenance phase (at target temperature) } \\
\hline$<12 \mathrm{~h}$ & $4.2 \%(1 / 24)$ \\
\hline $12-24 \mathrm{~h}$ & $58.3 \%(14 / 24)$ \\
\hline$>24 \mathrm{~h}$ & $37.5 \%(9 / 24)$ \\
\hline \multicolumn{2}{|l|}{ The use of cold fluids for induction of $\mathrm{TH}$} \\
\hline yes & $95.8 \%(23 / 24)$ \\
\hline no & $4.2 \%(1 / 24)$ \\
\hline \multicolumn{2}{|c|}{ The type of techniques used for maintaining hypothermia } \\
\hline $\begin{array}{l}\text { surface cooling without temperature feedback (ice } \\
\text { packs, cold pads) }\end{array}$ & $41.7 \%(10 / 24)$ \\
\hline surface cooling system with temperature feedback & $29.1 \%(7 / 24)$ \\
\hline $\begin{array}{l}\text { endovascular cooling system with temperature } \\
\text { feedback }\end{array}$ & $25 \%(6 / 24)$ \\
\hline $\begin{array}{l}\text { two techniques: surface and endovascular systems } \\
\text { with temperature feedback }\end{array}$ & $4.2 \%(1 / 24)$ \\
\hline \multicolumn{2}{|l|}{ The site for temperature monitoring } \\
\hline esophagus & $18.2 \%\left(4 / 22^{*}\right)$ \\
\hline bladder & $36.4 \%\left(8 / 22^{*}\right)$ \\
\hline concomitant esophagus and bladder & $45.4 \%\left(10 / 22^{*}\right)$ \\
\hline \multicolumn{2}{|l|}{ Qualifying ECG criteria for TH } \\
\hline any rhythm & $87.5 \%(21 / 24)$ \\
\hline only VF/VT & $12.5 \%(3 / 24)$ \\
\hline only PEA, asystole & $0 \%(0 / 24)$ \\
\hline \multicolumn{2}{|l|}{ Qualifying CA location criteria for TH } \\
\hline any place & $70.8 \%(17 / 24)$ \\
\hline prehospital CA & $16.7 \%(4 / 24)$ \\
\hline in-hospital CA & $12.5 \%(3 / 24)$ \\
\hline \multicolumn{2}{|l|}{ Use of neuromuscular-blocking agents } \\
\hline routinely & $41.7 \%(10 / 24)$ \\
\hline only when muscle shivering occur & $58.3 \%(14 / 24)$ \\
\hline
\end{tabular}

CA - cardiac arrest; PEA — pulseless electrical activity; $\mathrm{TH}$ - therapeutic hypothermia; VF — ventricular fibrillation; VT — ventricular tachycardia;

* - missing data $(n=2)$.

of dedicated cooling devices with temperature feedback (7\% in 2010 vs $20 \%$ in 2014). The use of temperature probes connected to the cooling device - a controlled feedback system - allows for continuous measurements of the patient's temperature and the adjustment of cooling elements accordingly. This approach is associated with increased safety and survival. ${ }^{12,13}$ 
Table 2. Barriers for wider deployment of TH in Poland - answers from units that do not perform $\mathrm{TH}(\mathrm{n}=54)$

\section{Does your unit plan to introduce TH in the future?}

\begin{tabular}{|l|c|}
\hline yes & $33 \%(18 / 54)$ \\
\hline no & $67 \%(36 / 54)$ \\
\hline \multicolumn{2}{|c|}{ What are the main obstacles to implementation of TH in the unit? } \\
\hline lack of knowledge, experience & $37 \%(20 / 54)$ \\
\hline lack of equipment & $20 \%(11 / 54)$ \\
\hline lack of reimbursement & $17 \%(9 / 54)$ \\
\hline lack of local protocol & $6 \%(3 / 54)$ \\
\hline lack of confidence in method & $4 \%(2 / 54)$ \\
\hline lack of medical staff & $2 \%(1 / 54)$ \\
\hline$>3$ of mentioned above & $15 \%(8 / 54)$ \\
\hline
\end{tabular}

$\mathrm{TH}$ - therapeutic hypothermia.

Table 3. Participation of units in dedicated therapeutic hypothermia training

\section{Did your center participate in a TH training course?}

\section{yes}

no

Would your center be willing to participate in a dedicated

TH training course (only centres that have not taken part in a training so far, $n=34$ )

yes

$68 \%(23 / 34)$

no
Are you aware of the available TH training opportunities? (centres willing to take part in a TH training, $n=23$ ) yes

no
$4 \%(1 / 23)$ $96 \%(22 / 23)$
The duration of active cooling in the present study was $12-24 \mathrm{~h}$ in $58.4 \%(14 / 24)$ of centers, $37.5 \%(9 / 24)$ of centers cooled for more than $24 \mathrm{~h}$ and only $4.2 \%(1 / 20)$ used $\mathrm{TH}$ for less than $12 \mathrm{~h}$. Although the current recommendations support $24 \mathrm{~h}$ of active cooling, there is some weak evidence to extend this time to $36 \mathrm{~h} .{ }^{14}$ Likewise, the value of the target temperature is becoming a hot topic for discussion, given the recent results by Nielsen et al., who showed no benefit of $33^{\circ}$ or $36^{\circ} \mathrm{C} . .^{15}$ We identified 1 cardiology center in Poland that conducts the target temperature management at $36^{\circ} \mathrm{C}$.

The main barrier for not using $\mathrm{TH}$ by cardiologists in Poland is the lack of knowledge and experience, which is an obstacle for $37 \%$ of cardiology centers. Financial restrictions were raised by $17 \%$ of centers, which is comparable to that reported by other European countries, i.e., Austria and the Czech Republic. ${ }^{16,17}$ However, given that the reimbursement of therapeutic hypothermia is available to each and every CICU, and there are flexible modes for renting the TH equipment, this problem is probably more an issue of local awareness than actual financial constraints. In fact, access to the equipment is not per- ceived as a major problem, with only $7.7 \%$ of units indicating that it prevents them from running a TH program for comatose post-CA survivors.

After the above-mentioned published randomized trial by Nielsen et al. that compared 2 target temperature strategies $\left(33^{\circ}\right.$ vs $\left.36^{\circ} \mathrm{C}\right)$, the view on the role of therapeutic hypothermia in post-resuscitation care has been extensively discussed. ${ }^{15}$ The most recent "Guidelines for Resuscitation 2015" published by the European Resuscitation Council include an option to use the target temperature of $36^{\circ} \mathrm{C}$ instead of the previously recommended $32-34^{\circ} \mathrm{C}$. As the present study describes experience before the year 2015, we have intentionally maintained the term $\mathrm{TH}$. However, it should be underlined that in order to comply with the current evidence and guideline terminology, future methodology should implement the notion of target temperature management.

The major limitation of our study was a low response rate (53\%) from CICUs. However, publication of the current results might in the future encourage other units to take part in a study and, more importantly, to start using $\mathrm{TH}$ in their wards. A direct comparison of anesthesiology units and cardiology units might be misleading as they differ in terms of patient profiles and indications for cooling. Nonetheless, one would expect that both types of units should have much higher rates of $\mathrm{TH}$ availability than is currently reported. The questionnaire used in the study had not been validated previously, therefore the results obtained should be interpreted causally. Nevertheless, we encourage authors of future studies to consider using this tool, which might provide additional date to validate it.

\section{Conclusions}

The use of TH in comatose patients after CA with ROSC is low. Scientific evidence supports the use of TH in this population to improve prognosis and quality of life. Financial restrictions have been eliminated recently, which could improve the adherence to the guidelines which recommend using $\mathrm{TH}$ in such patients. Therefore, a lack of knowledge and experience remain the greatest barriers for wide deployment of $\mathrm{TH}$. There is an urgent need to expand education and training among cardiologists, provided by centers experienced in $\mathrm{TH}$.

\section{References}

1. Skrifvars MB, Varghese B, Parr MJ. Survival and outcome prediction using the Apache III and the out-of-hospital cardiac arrest (OHCA) score in patients treated in the intensive care unit (ICU) following out-of-hospital, in-hospital or ICU cardiac arrest. Resuscitation.2012;83:728-733.

2. Belliard G, Catez E, Charron C, et al. Efficacy of therapeutic hypothermia after out-of-hospital cardiac arrest due to ventricular fibrillation. Resuscitation. 2007;75:252-259.

3. Bernard S, Gray T, Buist M, et al. Treatment of comatose survivors of out-of-hospital cardiac arrest with induced hypothermia. N Engl J Med. 2002;346:557-563. 
4. Hypothermia after Cardiac Arrest Study Group. Mild therapeutic hypothermia to improve the neurologic outcome after cardiac arrest. N Engl J Med. 2002;346:549-556.

5. Steg PG, James SK, Atar D, et al. ESC guidelines for the management of acute myocardial infarction in patients presenting with STsegment elevation: The task force on the management of ST-segment elevation acute myocardial infarction of the European Society of Cardiology (ESC). Eur Heart J. 2012;33:2569-2619.

6. Nolan JP, Soar J, Cariou A, et al. European Resuscitation Council and European Society of Intensive Care Medicine Guidelines for Post-resuscitation Care 2015: Section 5 of the European Resuscitation Council Guidelines for Resuscitation 2015. Resuscitation. 2015;95:202-222.

7. Krawczyk P, Kołodziej G, Szpyra B, Andres J. Implementation of therapeutic hypothermia after cardiac arrest in intensive care units in Poland. Kardiol Pol. 2013;71:270-274.

8. Kozinski M, Pstragowski K, Kubica JM, et al. ACS network-based implementation of therapeutic hypothermia for the treatment of comatose out-of-hospital cardiac arrest survivors improves clinical outcomes: The first European experience. Scand J Trauma Resusc Emerg Med. 2013;21:22.

9. Kowalik R, Szczerba E, Kołtowski $Ł$, et al. Cardiac arrest survivors treated with or without mild therapeutic hypothermia: Performance status and quality of life assessment. Scand J Trauma Resusc Emerg Med. 2014;22:76.

10. Zawiślak B, Depukat R, Arif S, Dudek D. Mild induced hypothermia in patient with acute myocardial infarction after cardiac arrest. Kardiol Pol. 2013;71:426-428.

11. Szymanski F, Karpinski G, Platek A, Puchalski B, Filipiak K. Long QT interval in a patient after out-of-hospital cardiac arrest with hypocalcemia, undergoing therapeutic hypothermia. Am J Emerg Med. 2013;31:1722 e1721-1723. doi: 10.1016/j.ajem.2013.07.026.

12. Kim JJ, Yang HJ, Lim YS, et al. Effectiveness of each target body temperature during therapeutic hypothermia after cardiac arrest. Am J Emerg Med. 2011;29:148-154.

13. Shin J, Kim J, Song K, Kwak Y. Core temperature measurement in therapeutic hypothermia according to different phases: Comparison of bladder, rectal, and tympanic versus pulmonary artery methods. Resuscitation. 2013;84:810-817.

14. Bisschops L, van der Hoeven J, Mollnes T, Hoedemaekers C. Seventy-two hours of mild hypothermia after cardiac arrest is associated with a lowered inflammatory response during rewarming in a prospective observational study. Crit Care. 2014;18:546.

15. Nielsen N, Wetterslev J, Cronberg T, et al. Targeted temperature management at 33 degrees $C$ versus 36 degrees $C$ after cardiac arrest. N Engl J Med. 2013;369:2197-2206.

16. Kliegel A, Gamper G, Mayr H. Therapeutic hypothermia after cardiac arrest in Lower Austria - a cross-sectional survey. Eur J Emerg 2011;18:105-107.

17. Skulec R, Truhlář A, Seblová J, et al. Implementation of pre-hospital therapeutic hypothermia in post-cardiac arrest patients in the Czech Republic. Resuscitation. 2012;83:e21-22.

\section{Supplement 1 \\ Implementation of therapeutic \\ hypothermia in post-cardiac arrest \\ patients treated in cardiac intensive care and coronary care units.}

\section{Contact details:}

I. Centre name:

II. Address:

III. Type:
a. cardiac intensive care unit (CICU)
b. coronary care unit $(\mathrm{CCU})$

IV. Name of the contact person:

\section{Questions:}

1. How many post-cardiac arrest patients (CA) were treated at your centre in recent year?

Number:

2. Is your centre using therapeutic hypothermia $(\mathrm{TH})$ in post-CA patients?
a. Yes
b. No

3. How many patients were treated with TH last year? Number:

4. How many patients treated with TH survived until discharge?

Number:

5. Which unit is treating post-CA patients at your center?
a. cardiac intensive care unit (CICU)
b. coronary care unit $(\mathrm{CCU})$
c. intensive care unit (ICU)

6. What is the target temperature in your protocol?
a. $32-34^{\circ} \mathrm{C}$
b. $<32^{\circ} \mathrm{C}$
c. $>34^{\circ} \mathrm{C}$

7. What is the cooling time in your protocol?
a. $<12 \mathrm{~h}$
b. $12-24 \mathrm{~h}$
c. $>24 \mathrm{~h}$

8. Do you use cold fluids for the inductions of TH?
a. Yes
b. No

9. What cooling methods do you use for the TH procedure?

a. Surface cooling with temperature feedback

b. Surface cooling without temperature feedback (ice, cold pads)

c. Intravascular cooling with temperature feedback

10. Site for temperature measurement?
a. Esophageal
b. Bladder
c. Rectal
d. At least 2 of the above (which?) 
11. Which primary rhythms leading to CA are included in $\mathrm{TH}$ procedure?

a. VT/VF

b. PEA, asystole

c. Both above

12. Inclusion for $\mathrm{TH}$ depending on the setting of $\mathrm{CA}$ :
a. Pre-hospital CA
b. In-hospital CA
c. Both settings

13. Is there a $\mathrm{TH}$ protocol in place at your center?

14. Do you use muscle relaxants for $\mathrm{TH}$ :
a. Yes, routinely
b. Only in case of muscle shivering

15. Do you plan to implement $\mathrm{TH}$ in the future (only if $\mathrm{TH}$ is not yet used)
a. Yes
b. No
c. $\mathrm{N} / \mathrm{a}$

16. What is the barrier for implementation of $\mathrm{TH}$ at your centre? (only if TH is not yet used)
a. Lack of knowledge, experience
b. Lack of reimbursement
c. Lack of TH protocol
d. Limited medical staff
e. Lack of TH equipment
f. Not convinced with the current body of evidence for the $\mathrm{TH}$ method
g. Other (which?)

17. Have you participated in TH Training?
a. Yes
b. No

18. Are you willing to take part in a TH Training? (only centers that have not participated in a training)
a. Yes
b. No

19. Do you know that TH Training is available and where to find it?

a. Yes,

b. No

20. If you are not willing to answer this questionnaire please share the reason why.

Thank you! 